\title{
PROSPECTS OF LINEAR MODEL PREDICTIVE CONTROL ON A 10 MW FLOATING WIND TURBINE
}

\author{
Frank Lemmer, Steffen Raach, David Schlipf, Po Wen Cheng \\ Stuttgart Wind Energy \\ Institute of Aircraft Design \\ University of Stuttgart \\ D-70569 Stuttgart/Germany \\ Email: \{lemmer,raach,schlipf,cheng\}@ifb.uni-stuttgart.de
}

\begin{abstract}
The presented research has the objective of supporting the integrated conceptual design of floating offshore wind turbines (FOWT). The dynamics of the multidisciplinary coupled system with the aerodynamics, hydrodynamics, structural dynamics, the catenary mooring lines and the controller shall be represented in simulation models adapted to the current design stage. Here, a linear model-predictive controller (MPC) as an optimal multiple input-multiple output (MIMO) controller is designed for a novel concept of the floating foundation for a $10 \mathrm{MW}$ wind turbine. The performance of this controller is easily adjustable by a cost function with multiple objectives. Therefore, the MPC can be seen as a benchmark controller in the concept phase, based on a simplified coupled simulation model with only approximate model information. The linear model is verified against its nonlinear counterpart and the performance of the MPC compared to a SISO PI-controller, which is also designed in this work. The developed models show to be well suited and the linear MPC shows a reduction of the rotor speed overshoot and tower bending from a deterministic gust.
\end{abstract}

\section{Introduction}

Offshore wind power is considered to play a favorable role in the renewable power production. High and more constant wind speeds help to guarantee a stable power production. The limitations of fixed-bottom solutions regarding steepness of the coasts and water depth can be overcome by using floating foundations for offshore wind turbines. Research on floating offshore wind turbines (FOWT) has advanced appreciably during the last ten years. Experience and modeling techniques have been widely adopted from oil and gas industry and adjusted to the specific needs of wind power conversion. While a common horizontal axis wind turbine is mostly mounted on the floating structure research has not agreed yet on a specific concept of the floating platform. Spar-type platforms seem well suited and have already been tested in full-scale. The large draft yields a high restoring moment to withstand the aerodynamic thrust force and the viscous damping from flow separation improves the system dynamics. However, more and more existing prototypes are semisubmersibles. Their low draft implies that the concept might get a larger share on the market. A drawback of these types is that the structure mostly requires an expensive welded steel structure to produce the required restoring moment. As a consequence concrete as structural material has been considered for floating wind turbines already, see [1].

For this work a concrete spar-type concept has been designed for a $10 \mathrm{MW}$ wind turbine. The floating structure has comparable material costs as a steel structure but it has the advantage of a longer lifetime than the steel counterpart. So far, parametrized conceptual calculations of hydrostatics, still-water hydrodynamics and wave excitation forces have been done for the design. The mooring system is not yet designed and the viscous hydrodynamic forces have not yet been identified.

The implementation of an MPC at this early design stage shall point out the advantages of an integrated design methodology from the beginning. The controller has a significant impact on the system dynamics. The contradicting goals of stabilizing power for above-rated wind speeds and minimizing platform motion are a key challenge, as has been reported repeatedly in the literature. A too aggressively tuned blade-pitch controller results in unstable platform behavior. This is due to a non-negative phase zero of floating platforms, which limits the bandwidth of the blade pitch controller. A nice explanation of this "negative 
damping" problem is given in [2]. For an uncoupled adaptation of the controller the bandwidth of the PI-controller can be reduced in order not the excite the platform modes, see [3]. Another method for a de-coupled tuning of the gains is given in [4]. The different methods have been applied and compared by [5]. Another application of these control design methods to existing concepts with coupled models is given by [6] and by the author of this paper under his former name Sandner, see [7]. As mentioned before, two control goals hold at the same time and therefore MIMO strategies have been investigated. First, with only one actuated variable as the blade pitch angle and conventional SISO controller design, see [8] but also with additional actuators to stabilize the tower have been implemented in [9]. Other multivariable controllers have been developed by [10] and [11]. An extensive study on individual pitch control (IPC) for floating wind turbines can be found in the thesis [12]. Recently, also the inclusion of disturbance preview for floating wind turbines has been studied. A nonlinear model-predictive controller (NMPC) with Lidar (Light detection and ranging) wind measurements is presented in [13] and extended for IPC in [14]. A model development and linear model predictive control design can be found in [15].

In this paper, a linear MPC, which is an optimal controller, is developed as an approach for an easily tunable controller for a new conceptual platform. First, the considered design of a concrete floating platform with a $10 \mathrm{MW}$ wind turbine is presented before the simplified dynamic simulation models are introduced for the subsequent design of the baseline PI-controller and the MPC. Finally, the simulation results are presented and discussed.

\section{Design description}

The conceptual concrete floating platform design subject to this study can be classified as a spar with modifications to allow a reduction of the overall draft. This makes the concept more flexible to the site and opens markets of shallow coastlines such as those appearing widely in Europe. A spar-type floater gains the restoring moment and stability through a center of gravity at a low position. A large metacentric height of a lightweight platform can achieve enough restoring for resisting the thrust forces of a 10MW wind turbine. Such a design would be a very slender cylinder of a draft of more than $150 \mathrm{~m}$. The low material cost of concrete and the requirement to use simple shapes, which holds for concrete structures, leads to a spar-type platform. The toroidal shape allows a reduction of the draft combined with an increase of the radius while ensuring limited vertical wave excitation forces (Froude-Krylov forces). The interface between the concrete torus and the tower base is realized with four steel struts for a spatial introduction of the sectional forces to the concrete body. The torus is always fully submerged with the upper torus surface at a depth of $10 \mathrm{~m}$. The heave plates at the lower part

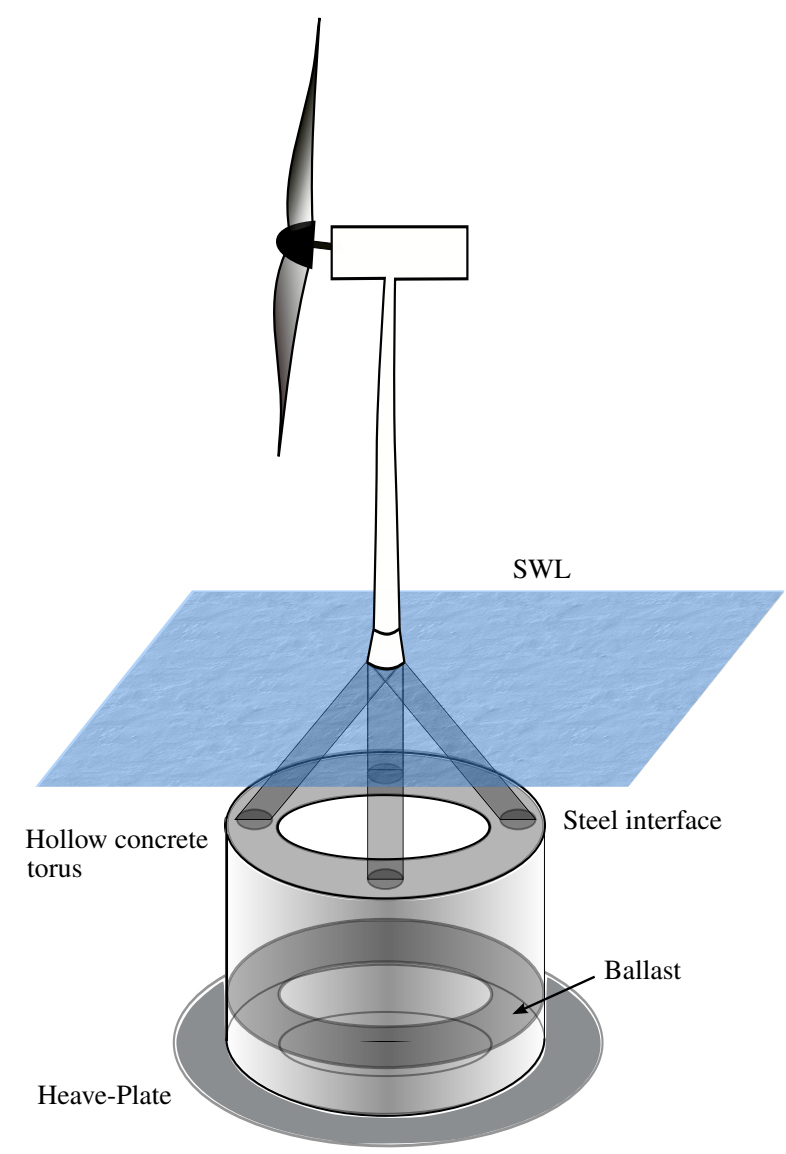

FIGURE 1: Sketch of concrete torus platform.

of the platform are not included yet but considered a feature for detailed tuning of the hydrodynamic properties in later design stages. Also the mooring lines are not designed yet but represented through a horizontal linear stiffness at the overall center of mass, see Table 2. The unidirectional spring yields a natural period in surge of $T_{e i g, 11} \approx 250 \mathrm{~s}$, which is realistic for the platform mass and a catenary mooring system. The application at the overall center of mass shall not alter the dynamics significantly, except for the restoring force in $x$-direction for this design stage.

The wind turbine is the generic 10MW INNWIND.EU reference wind turbine, see [16], which has been designed and published for research on large offshore wind turbines. The design is made for offshore applications on a jacket foundation. Therefore, the controller could not be adapted for this work since the foundation dynamics are not comparable. The main dimensions of the concept together with the $10 \mathrm{MW}$ reference wind turbine is shown in Table 1. 
TABLE 1: FOWT design parameters.

\begin{tabular}{lll}
\hline Platform: & Draft [m] & 58.7 \\
& Outer diameter [m] & 22.0 \\
& Inner diameter [m] & 15.0 \\
& Reinforced concrete \& & $1.489 \times 10^{7}$ \\
& steel interface mass [kg] & \\
& Ballast mass [kg] & $2.58 \times 10^{7}$ \\
& Platform center of mass & 44.98 \\
& below SWL [m] & \\
& Metacentric height [m] & 4.205 \\
Wind turbine: & Rating [MW] & 10.0 \\
& Rated wind speed [m/s] & 11.4 \\
& Rated rotor speed [rpm] & 9.6 \\
& Optimal tip speed & 7.86 \\
& ratio (TSR) [-] & 178.3 \\
& Rotor diameter [m] & 119.0 \\
Hub height [m] & Rotor-nacelle assembly & $8.84 \times 10^{5}$ \\
mass [kg] & \\
\hline
\end{tabular}

\section{Modeling}

Numerous coupled modeling techniques with different levels of detail have been presented in the literature. State-of-theart simulation models for load case simulations, applicable for certification consist of multibody systems (MBS) with bladeelement momentum theory (BEM) for the rotor aerodynamics and for the hydrodynamics the pre-processed frequency-domain results of the radiation and the diffraction problem, respectively, see [17] and [18]. These are then transformed into time domain, also accounting for radiation memory effects. The mooring lines are mostly modeled through quasi-static force-displacement relations but also dynamic models are in use. For control-design, however, simplified models are necessary, which have to be selected and designed carefully in order to neglect all but the relevant effects. Such models have been designed and compared to verified models, see, e.g., [19] and [20].

Here, a simplified multibody model, initially presented in [21] and verified in [22] has been adapted for the new system.

\subsection{Nonlinear model}

The nonlinear equations of motion which are needed for the design and verification of the controllers are set up for a multibody system of rigid bodies applying the Newton-Euler equations. The model set up for this work has four degrees of freedom (DOFs) with the generalized coordinates platform surge $x_{p}$ and pitch $\beta_{p}$ motion, the tower top displacement in platform co- ordinates, $x_{t}$ and the rotor azimuth angle $\varphi$ comprised in $\mathbf{q}$ as

$$
\mathbf{q}=\left[\begin{array}{c}
x_{p} \\
\beta_{p} \\
x_{t} \\
\varphi
\end{array}\right]
$$

The considered rigid bodies are the Platform, the Tower, the $\mathrm{Na}$ celle and the Rotor, see Figure 2.

To set up the Newton-Euler equations following [23] Newton's second law for translational motion is written for each body as well as Euler's law for rotational motion. The coupled equations of motion result for translational and rotational directions for each body of the $p$ bodies with mass $m_{i}$ and inertia tensor $\mathbf{I}_{i}$ as

$$
\left[\begin{array}{c}
m_{i} \mathbf{E} \cdot \mathbf{J}_{T i} \\
\vdots \\
\mathbf{I}_{i} \cdot \mathbf{J}_{R i} \\
\vdots
\end{array}\right] \ddot{\mathbf{q}}+\left[\begin{array}{c}
m_{i} \mathbf{E} \cdot \overline{\mathbf{a}}_{i} \\
\vdots \\
\mathbf{I}_{i} \cdot \bar{\alpha}_{i}+\tilde{\omega}_{i} \mathbf{I}_{i} \omega_{i} \\
\vdots
\end{array}\right]=\left[\begin{array}{c}
\mathbf{f}_{i} \\
\vdots \\
\mathbf{l}_{i} \\
\vdots
\end{array}\right]+\overline{\mathbf{Q}} \cdot \mathbf{g} .
$$

Equation (2) has the dimension of $2 \cdot 3 \cdot p$, which is Newton's and Euler's law in each spatial direction for each body $i$. The kinematics are derived in a convenient manner for an automated setup of the equations by applying Jacobian matrices, which transform the generalized velocities to inertial coordinates. The translational Jacobian matrices $\mathbf{J}_{T i}$ and the rotational Jacobian matrices $\mathbf{J}_{R i}$ are with each body's position vector $\mathbf{r}_{i}$ and each body's angular velocity vector $\omega_{i}$ defined as

$$
\mathbf{J}_{T i}=\frac{\partial \mathbf{r}_{i}}{\partial \mathbf{q}} \quad \omega_{i}=\mathbf{J}_{R i}(\mathbf{q}) \cdot \dot{\mathbf{q}}
$$

The second term of Eq. (2) is the vector of Coriolis-, centrifugal and gyroscopic forces and moments with the generalized accelerations $\ddot{\mathbf{q}}$ and the local translational and rotational accelerations $\overline{\mathbf{a}}_{i}$ and $\bar{\alpha}_{i}$ due to the accelerations of each body's reference frame. They are calculated for scleronomic systems as

$$
\overline{\mathbf{a}}_{i}=\dot{\mathbf{J}}_{T i} \dot{\mathbf{q}} \quad \bar{\alpha}_{i}=\dot{\mathbf{J}}_{R i} \dot{\mathbf{q}} .
$$

The term including the skew-symmetric matrix of each body's angular velocity vector $\tilde{\omega}_{i}$ results from the time derivative of the rotation matrix that transforms the local mass moment of inertia matrices $\mathbf{I}_{i}$ of each body $i$ into global coordinates. On the right hand side of Eq. (2) are all applied forces and moments $\mathbf{f}_{i}$ 


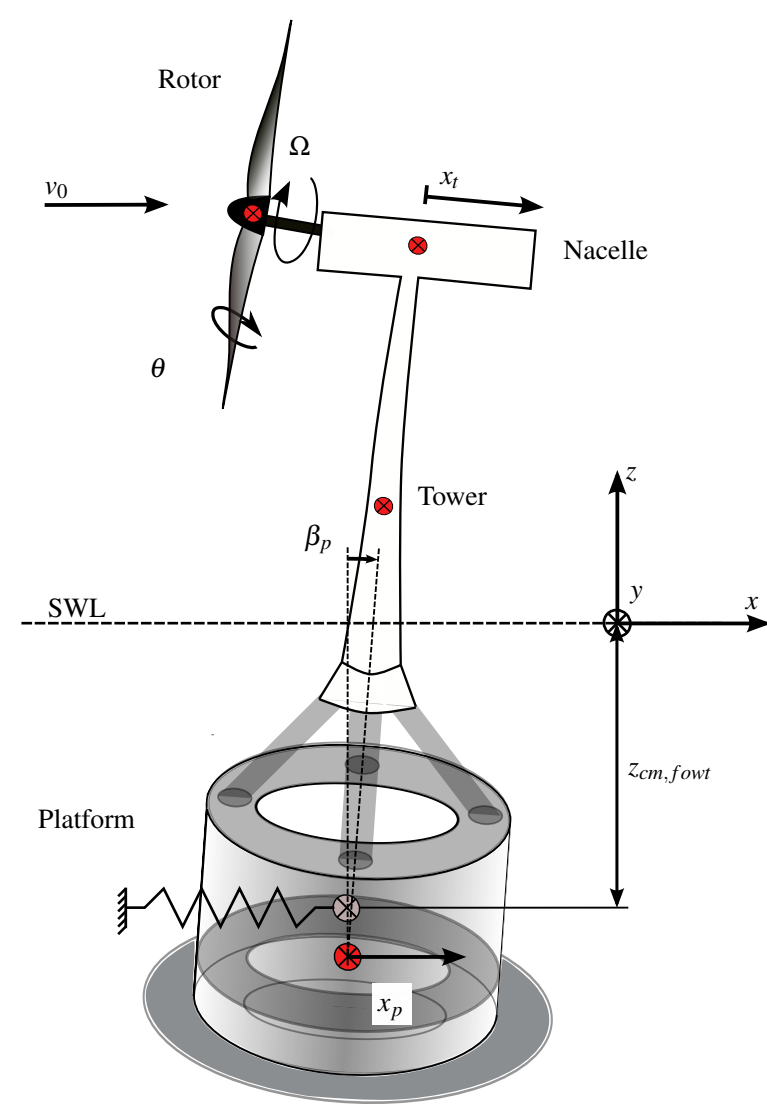

FIGURE 2: Multibody system sketch.

and $\mathbf{l}_{i}$, respectively and the reaction forces $\overline{\mathbf{Q}} \cdot \mathbf{g}$. These forces point always into constrained directions and are eliminated in a next step, according the d'Alembert's principle, through a multiplication of Eq. (2) by the inverse global jacobian matrix $\mathbf{J}$ from the left.

Finally, a state space description can be derived from Eq. (2). This is symbolically written in C-code and simulated with a fourth order fixed-step Runge-Kutta solver. With the transformed equation (2) the new variables result as the mass matrix $\mathbf{M}$, the vector of applied forces and moments $\mathbf{p}$ and the Coriolis-, centifugal and gyroscopic forces $\mathbf{k}$. The first derivative of the state vector reads as

$$
\dot{\mathbf{x}}=\frac{\partial \mathbf{x}}{\partial t}=\left[\begin{array}{c}
\dot{\mathbf{q}} \\
\ddot{\mathbf{q}}
\end{array}\right]=\left[\begin{array}{c}
\dot{\mathbf{q}} \\
\mathbf{M}^{-1}(\mathbf{p}-\mathbf{k})
\end{array}\right]
$$

The hydrodynamic forces as part of the external force vector $\mathbf{p}$ are reduced to the still water hydrodynamics. The hydrostatic coefficients are linearized and constant. For the added mass coefficients calculations have been done with Ansys Aqwa and the infinite-frequency limit of the added mass matrix $\mathbf{A}$ is used

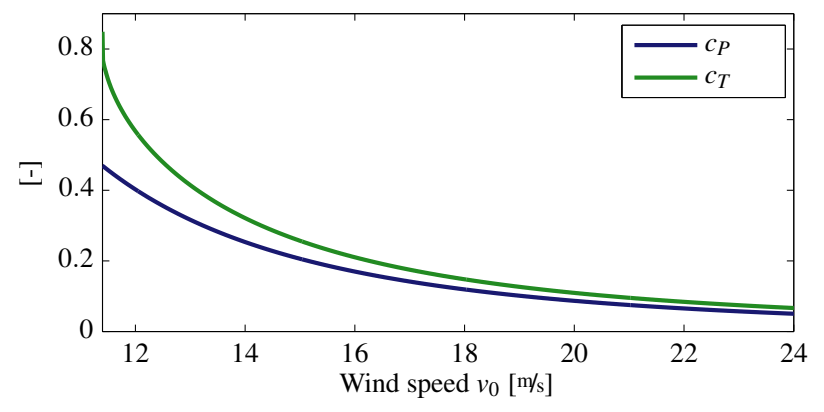

FIGURE 3: Steady-state power and thrust coefficients $c_{P}$ and $c_{T}$ over wind speeds $v_{0}$ for $10 \mathrm{MW}$ reference wind turbine.

for these coupled simulations. The hydrodynamic viscous damping has been approximated with the values given in Table 2.

The aerodynamic forces and moments within $\mathbf{p}$ are calculated based on the steady-state power and thrust coefficients $c_{P}(T S R, \theta)$ and $c_{T}(T S R, \theta)$ over blade pitch angles $\theta$ and tip-speed ratios $T S R$ of the $10 \mathrm{MW}$ reference wind turbine. The aerodynamic torque is calculated by

$$
M_{a}=\frac{1}{2} \rho \pi R^{3} \frac{c_{P}(T S R, \theta)}{T S R} v_{r e l}^{2}
$$

with the rotor radius $R$ and the relative rotor-effective wind speed $v_{r e l}$, see [25]. The aerodynamic thrust force in shaft direction reads as

$$
F_{a}=\frac{1}{2} \rho \pi R^{2} c_{T}(T S R, \theta) v_{r e l}^{2}
$$

The steady-state power and thrust coefficients $c_{P}$ and $c_{T}$ can be written as function of $T S R$ and blade pitch angle $\theta$. In Figure 3 they are plotted over the wind speeds $v_{0}$ for the steady-state blade pitch angles $\theta$. The power coefficient $c_{P}$ decreases for increasing wind speeds, as well as its derivative $\frac{\partial c_{P}}{\partial v}$, which is the main reason for the common implementation of gain scheduling. The gain scheduling keeps the selected properties of the closed loop drivetrain dynamics constant over all operational wind speeds, see Section 4. In the coupled floating case, also the thrust coefficient behavior over wind speeds $v_{0}$ is important. It shows an even higher slope over $v_{0}$ as the power coefficient $c_{P}$.

For the blade pitch actuator and additional second order dynamic has been included with a natural frequency $\omega_{0, \text { act }}=1.6 \mathrm{~Hz}$ and a damping ratio $\xi_{\text {act }}=0.8$.

\subsection{Linear model}

A linear model will now be developed from the nonlinear equations of motion for the design of the PI-controller and for use 
TABLE 2: Hydrodynamic platform properties about platform center of mass.

\begin{tabular}{llll}
\hline $\begin{array}{l}\text { Hydrostatic stiffness } \\
\text { (including gravitation) }\end{array}$ & $C_{55}$ & {$[\mathrm{Nm} / \mathrm{rad}]$} & $2.29 \times 10^{9}$ \\
Added mass & $A_{11}$ & {$[\mathrm{~kg}]$} & $1.0 \times 10^{7}$ \\
Added mass & $A_{55}$ & {$\left[\mathrm{kgm}^{2}\right]$} & $1.3 \times 10^{10}$ \\
Added mass & $A_{15}$ & {$[\mathrm{kgm}]$} & $5.0 \times 10^{8}$ \\
Added mass & $A_{51}$ & {$[\mathrm{kgm}]$} & $5.0 \times 10^{8}$ \\
Damping coefficient & $d_{11}$ & {$[\mathrm{Ns} / \mathrm{m}]$} & $2.277 \times 10^{5}$ \\
Damping coefficient & $d_{55}$ & {$[\mathrm{Nms} / \mathrm{rad}]$} & $1.27 \times 10^{9}$ \\
Damping ratio & $\xi_{11}$ & {$[-]$} & 0.114 \\
Damping ratio & $\xi_{55}$ & {$[-]$} & 0.0023 \\
Linear mooring stiffness & $C_{l, 11}$ & {$[\mathrm{~N} / \mathrm{m}]$} & $6.18 \times 10^{4}$ \\
Linear mooring stiffness & $C_{l, 55}$ & {$[\mathrm{Nm} / \mathrm{rad}]$} & 0.0 \\
Linear mooring stiffness & $C_{l, 15}$ & {$[\mathrm{~N} / \mathrm{rad}]$} & $6.18 \times 10^{4}$ \\
Linear mooring stiffness & $C_{l, 51}$ & {$[\mathrm{~N}]$} & 0.0 \\
\hline
\end{tabular}

as internal model of the MPC. Therefore, the nonlinear equations of motion of Eq. (5) are linearized, transforming the states $\mathbf{x}$ to the new differential state vector $\Delta \mathbf{x}$ as well as the inputs $\mathbf{u}$ to the differential input $\Delta \mathbf{u}$

$$
\mathbf{x}=\mathbf{x}_{0}+\Delta \mathbf{x} \quad \mathbf{u}=\mathbf{u}_{0}+\Delta \mathbf{u}
$$

Eventually, the position and velocity-dependent terms of the state-space description, Eq. (5) can be separated resulting in the linear state-space formulation

$$
\Delta \dot{\mathbf{x}}=\underbrace{\left[\begin{array}{cc}
\mathbf{0} & \mathbf{E} \\
-\mathbf{M}^{-1} \cdot \mathbf{Q}-\mathbf{M}^{-1} \cdot \mathbf{P}
\end{array}\right]}_{\mathbf{A}} \Delta \mathbf{x}+\mathbf{B} \cdot \Delta u .
$$

The highly nonlinear aerodynamic kinetics from Eqn. (6) and (7) part of $\mathbf{P}$ and $\mathbf{Q}$. They represented by a first order linearization with respect to the blade pitch angle $\theta$, the rotor speed $\Omega$ and the wind speed $v_{0}$. The linearized formulation of the thrust force $F_{a}$ and the torque $M_{a}$ is given in [7].

The dynamics of the open loop system can be seen in the Bode plot in Figure 4, where the input has been set to the blade pitch angle $\theta$ and the output to the rotor speed $\Omega$. For rated wind speed at $v_{0}=11.6 \mathrm{~m} / \mathrm{s}$ a large phase loss can be seen, which is less pronounced for higher wind speeds. An eigenanalysis reveals the modes and the corresponding damped eigenfrequencies $\omega_{d}$, shown in Table 3 . The modes are labelled according to their dominant DOF. For controller design of FOWT the right half-plane zeros (RHPZ) are of major importance as discussed in Chapter 1. All zeros of this open-loop system have a frequency $\omega_{z, i}$ close to an eigenvalue or pole, which can be seen

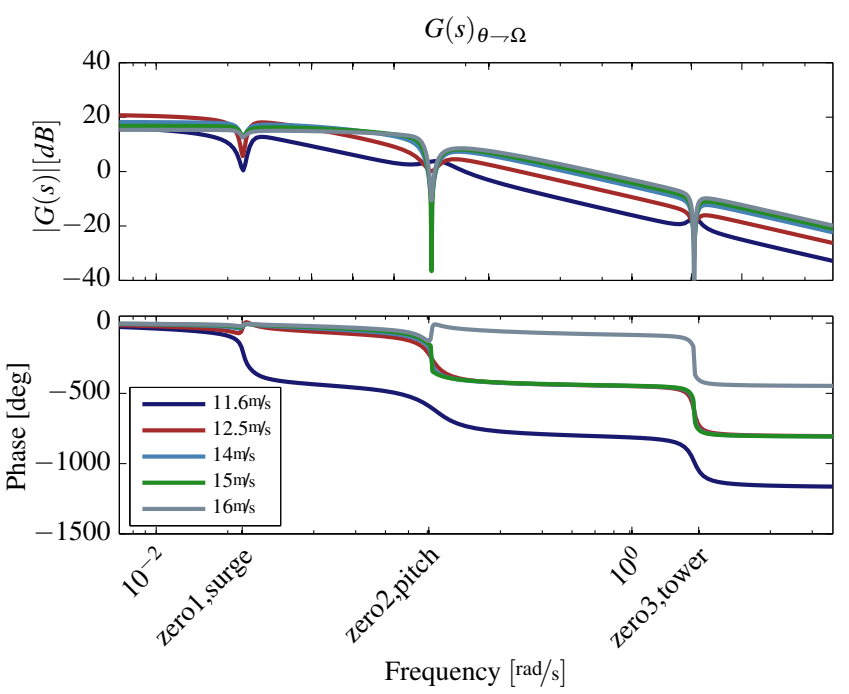

FIGURE 4: Bode diagram for the open loop from blade pitch angle $\theta$ to the rotor speed $\Omega$ for $v_{0}=11.6 \ldots 16 \mathrm{~m} / \mathrm{s}$.

\begin{tabular}{|c|c|c|c|c|c|c|c|c|}
\hline$x_{p}$ & 0.1 & 0.0 & 1.0 & 1.0 & 0.26 & 0.26 & 0.002 & 0.002 \\
\hline$\beta_{p}$ & 0.0 & 0.0 & 0.001 & 0.001 & 0.06 & 0.06 & 0.001 & 0.001 \\
\hline$x_{t}$ & 0.0 & 0.0 & 0.007 & 0.007 & 0.25 & 0.25 & 1.0 & 1.0 \\
\hline$\Omega$ & 1.0 & 1.0 & 0.4 & 1.0 & 1.0 & 1.0 & 0.007 & 0.007 \\
\hline $\begin{array}{c}\omega_{d, i} \\
{[\mathrm{rad} / \mathrm{s}]}\end{array}$ & 0.0 & 0.0 & 0.02 & 0.2 & 0.15 & 0.15 & 1.8 & 1.8 \\
\hline $\begin{array}{c}\omega_{z, i} \\
{[\mathrm{rad} / \mathrm{s}]}\end{array}$ & & 0.0 & 0.02 & 0.02 & 0.14 & 0.14 & 1.8 & 1.8 \\
\hline & $0^{80}$ & $0^{20}$ & $5^{0}$ & s. & $e^{-2}$ & $\hat{j}^{N}$ & $x^{0^{2}}$ & $e^{0^{2}}$ \\
\hline
\end{tabular}

TABLE 3: Results of open loop Eigenanalysis @ $v_{0}=12 \mathrm{~m} / \mathrm{s}$.

in the Bode plot, Fig. 4 and in Table 3. Here, two RHPZ appear at a period of $T_{\mathrm{RHPZ}, 1}=44.1 \mathrm{~s}$ and $T_{\mathrm{RHPZ}, 2}=3.4 \mathrm{~s}$, close to the periods of the pitch- and tower eigenfrequencies, respectively. These two modes are the ones which move visibly for changing feedback gains in the closed loop. This will be shown in the next section on the baseline and MPC controller design.

The validity of this model is shown in the next section, where time-domain simulations with the same disturbance and the same controller are performed with the linear and the nonlinear model.

\subsection{Model validation}

The ability of reduced models to predict the system dynamics of the real system has to be evaluated with respect to accuracy and state space of the reduced model. In the following, two extreme operating gusts around the wind speeds $v_{0}=[8,16] \mathrm{m} / \mathrm{s}$ are simulated with the nonlinear and the linear model to compare the 

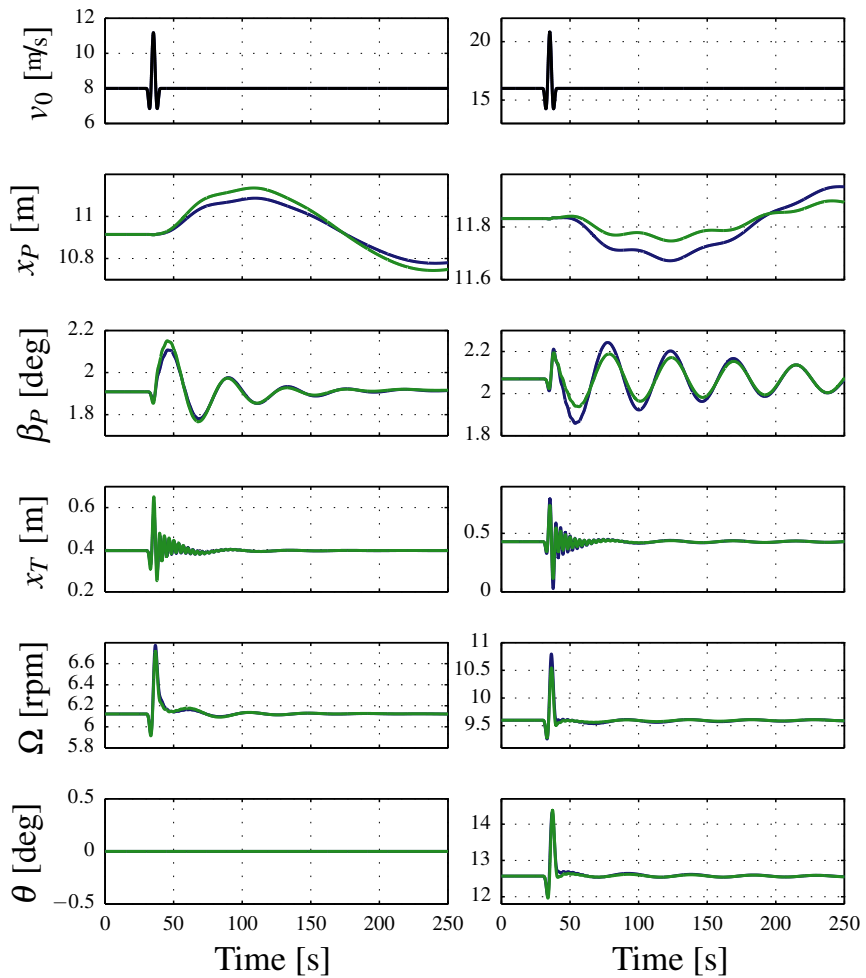

FIGURE 5: Nonlinear model (blue) vs. linear model (green) for $v_{0}=8$ (left) and $16 \mathrm{~m} / \mathrm{s}$ (right).

system dynamics. Figure 5 shows the platform DOFs $x_{P}$ and $\beta_{P}$, below the tower-top displacement $x_{T}$ and at the bottom the rotor speed $\Omega$ and the blade pitch angle $\theta$. While the latter agree very well for all wind speeds the platform surge and pitch displacements are overpredicted by the linear model for wind speeds below rated and underpredicted for wind speeds above rated. This is most likely due to the linearization of the aerodynamics, described in Section 3. Regarding the nonlinearity of the aerodynamic coefficients, however, it can be said that the frequency as well as the phase results of the two models agree rather well and thus, the linear model is regarded as valid for the purposes of this work.

\section{Controller}

In this section two controllers for the presented FOWT are developed, a baseline controller and a model predictive controller.

\subsection{Baseline controller}

The baseline controller follows the standard control strategy of wind turbines, which divides the area of operation into several

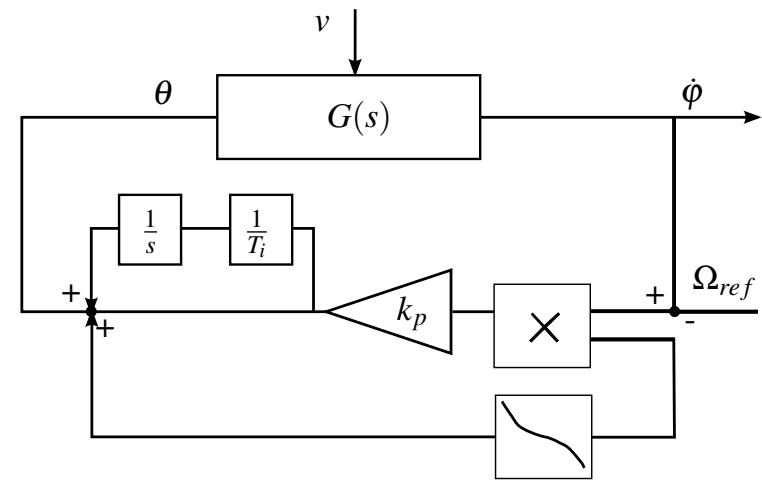

FIGURE 6: Block diagram of the pitch controller with the gain scheduling function.

parts with different controllers and control goals. Between the different regions transition strategies are included for a smooth switchover. The controller mainly consists of a torque controller and a pitch controller.

Region 2 controller The region 2 controller aims at maximizing power output by tracking the optimal tip speed ratio $\lambda_{\text {opt }}$ through the generator torque, while the blade pitch angle $\theta$ is constant. The tip speed ratio $T S R$ is defined by

$$
T S R=\frac{\Omega R}{v_{0}},
$$

where $\Omega$ is the rotor speed, $v_{0}$ the wind speed and $R$ the rotor radius. This is done as described in [24] by setting the generator torque to

$$
M_{g}=k \Omega^{2}
$$

with $k=\frac{1}{2} \rho \pi R^{3} \frac{c_{p, \max }}{T_{\text {SRopt }}{ }^{3}}$, where $\rho$ is the air density and $c_{p, \max }$ the maximum power coefficient. Between region 2 and region 3 a transition strategy applies, which is a linear interpolation between the generator torque $M_{g}$ in region 2 and $M_{g, \text { rated }}$.

Region 3 controller In region 3 the torque controller is set to be constant to $M_{g, \text { rated }}=\frac{P_{\text {rated }}}{\Omega_{\text {rated }}}$. This is done to stabilize the negative coupling between pitch controller and platform pitch motion as described in Chapter 1. The pitch controller is a standard PIcontroller for blade pitch control, with a PI-feedback of the rotor speed error. In Figure 6 the closed loop block diagram is shown.

Conventional SISO methods are applied using the developed linear coupled model. Therefore, the open and closed loop polezero map is plotted for various proportional and integral gains. The range of the proportional gains $k_{p}$ is selected such that the 
movement of the poles from the open loop pole towards the open loop zero is visible and also some platform poles with a positive real part result. As in [7] the underlying idea is that increasing proportional gains generally improve the performance of the rotor speed tracking, while they can lead to instability of the floating platform. Thus, a well suited controller can be designed by selecting stable closed loop platform poles, close to the imaginary axis, with their corresponding gains $k_{p}$ and $T_{i}$. This method for baseline controller design of FOWT assumes that with the described pole placement the compromise between rotor speed tracking and stable platform motion is close to the optimum. Subsequently, a nonlinear simulation model with realistic disturbances is used to find the optimal combination of proportional gain and integral gain time constant $T_{i}$.

Once a reasonable range of gains $k_{p}$ has been selected the pole-zero map shows clearly the poles, which move significantly with the proportional gains $k_{p}$. For the PI-controller developed here these modes $\lambda_{i}$ have been constrained to a reasonable value with a real part $0>\mathfrak{R e}\left(\lambda_{i}\right)>\mathfrak{R e}\left(\lambda_{O L}\right)$ for all wind speeds $v_{\text {rated }}<v_{0}<v_{\text {cutout }}$. Subsequently, all gains $k_{p}$ and time constants $T_{i}$ fulfilling the condition of the real part $\mathfrak{R e}\left(\lambda_{i}\right)$ give the design space for a further brute-force optimization under turbulent wind, simulated with the nonlinear model.

The pole-zero map is shown in Figure 7 for the dynamics from wind speed $v_{0}$ to rotor speed $\Omega$ in case of the closed-loop and from blade-pitch angle $\theta$ to rotor speed $\Omega$ in the open-loop case. The plot shows the poles and zeros with positive imaginary part for two different wind speeds $v_{0}=[11.6,15.0] \mathrm{m} / \mathrm{s}$ in the upper to plots. The third plot is a detail on the first wind speed. It can be seen how the tower mode and the platform pitch-mode move significantly to the right. As already mentioned in Chapter 3 these are the modes with associated zeros at neighboring frequencies. For low wind speeds, slightly above rated $(11.6 \mathrm{~m} / \mathrm{s})$ especially the platform pitch-mode moves to the right, whereas for higher wind speeds the tower-mode becomes the critical mode. The platform surge-pole, represented by the blue line in the third plot of Fig. 7, with no associated RHPZ does not move significantly for varying $k_{p}$. At rated wind speeds two RHPZ can be seen, whereas only one remains at $v_{0}=15 \mathrm{~m} / \mathrm{s}$, which explains why the tower-mode becomes critical for higher wind speeds. With the set limit of the real part of the closed loop poles for all wind speeds the stable combinations of the PIcontroller gains have been determined. Figure 8 shows how the proportional gains $k_{p}$, which satisfy the selected constraint on the real part $\mathfrak{R e}\left(\lambda_{i}\right)$, evolve over the wind speeds $v_{0}$.

Through nonlinear simulations the optimal set of controller gains $k_{p}$ and $T_{i}$ has been found in one-hour simulations at wind speeds $v_{0}=[15,18,20] \mathrm{m} / \mathrm{s}$. As a cost function the variance of the rotor speed $\Omega$ and the variance of the tower top displacement $x_{t}$ has been used. Eventually, the optimal time constant $T_{i}=40 \mathrm{~s}$ has been selected, see Figure 8. The nonlinear gain scheduling is realized with a lookup table.
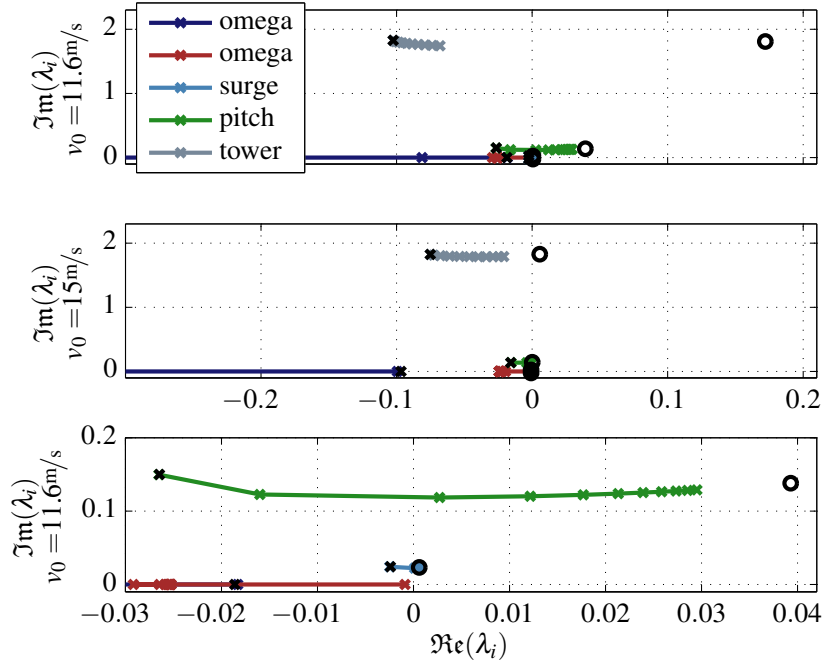

FIGURE 7: Pole-zero map for open loop (black) and closed loop for $k p=0.0001 \ldots 0.081$ and $T_{i}=40 \mathrm{~s}$, poles $(\times)$, zeros (o).

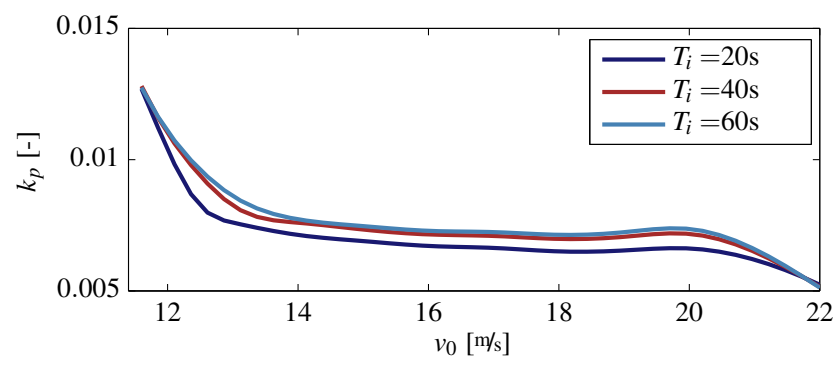

FIGURE 8: Proportional gains $k_{p}$ over wind speeds $v_{0}$ and time constants $T_{i}=20 \ldots 60 \mathrm{~s}$.

In a next step a model predictive controller is developed, based on a cost functional including the relevant dynamics, and analyzed for its applicability to improve the dynamics compared to the PI-controller.

\subsection{Model predictive controller}

The basic idea of model predictive control is to solve the optimal control problem, apply only a defined time span of the solution, measure the current states and solve the optimal control problem again with the measured states as initial condition. The feedback is induced by solving the optimal control problem repetitively. Furthermore, input and stage constraints can be taken into account as well as multi-input and multi-output (MIMO). The inputs are the commanded collective blade pitch angle $\theta_{c}$ and the generator torque $M_{g}, \boldsymbol{u}=\left[\theta_{c}, M_{g}\right]$.

Here, a linear model predictive control strategy is investi- 
gated. Therefore, the linearized model of Section 3.2 is used. The nonlinear state space is discretized with respect to the wind speed and for each operation point of the wind $v_{0, i}$ the linear system dynamics are calculated as described in Section 3.2. Thus, a set of linear systems

$$
\dot{\boldsymbol{x}}=\boldsymbol{A}\left(v_{0, i}\right) \boldsymbol{x}+\boldsymbol{B}\left(v_{0, i}\right) \boldsymbol{u}+\boldsymbol{B}_{d}\left(v_{0, i}\right) d
$$

are obtained, which were then used in the optimal control problem.

Thus, the considered optimal FOWT control problem can be described as follows. The objective is to find the optimal control trajectory $\boldsymbol{u}(\cdot)$ in the presence of the disturbance $\boldsymbol{d}(\cdot)$, minimizing the cost functional $\boldsymbol{J}$, which is defined over the time horizon $T$ of the objective function $\Pi$ from the actual time $t_{0}$ to the final time $t+T$ with the set of linear systems and the set of constraints $H$. The time horizon is discretized intro $N=10$ steps with a step size of $d t=0.2 \mathrm{~s}$. Further, a discretized version of Eq. (12) is used

$$
\boldsymbol{x}(k+1)=\boldsymbol{A}\left(v_{0, i}\right) \boldsymbol{x}(k)+\boldsymbol{B}\left(v_{0, i}\right) \boldsymbol{u}(k)+\boldsymbol{B}_{d}\left(v_{0, i}\right) d(k) .
$$

Then, the optimal control problem can be stated as follows:

$$
\begin{array}{cl} 
& \min _{\boldsymbol{u}} \boldsymbol{J}(\boldsymbol{x}, \boldsymbol{u}, d) \\
\text { with } & \boldsymbol{J}(\boldsymbol{x}, \boldsymbol{u}, d)=\sum_{k=1}^{N} \Pi(\boldsymbol{x}(k), u(k-1))+\Pi_{\infty} \\
\text { s.t. } & \boldsymbol{x}(k+1)=\boldsymbol{A} \boldsymbol{x}(k)+\boldsymbol{B} \boldsymbol{u}(k)+\boldsymbol{B}_{d} d(k) \\
& \boldsymbol{x}(0)=x_{0} \\
& H(\boldsymbol{x}, \boldsymbol{u}, d) \geq \boldsymbol{0} \forall k=1 \ldots N .
\end{array}
$$

The system dynamics are chosen with respect to the current wind speed, which can be calculated from the current states by solving the observer equation for the wind speed, see [25]. For the preview on the rotor effective wind speed $v_{0}$ the wind speed is kept constant to the actual wind speed.

The crucial part in designing a model predictive controller is to translate the control goals to a representative mathematical expression. Generally, the goal in wind turbine control is to maximize power output within the area of operation and to minimize structural loads. Further, damping the platform motion of the FOWT is also an objective.

Above rated wind speed control The main control goal in above rated control is to keep constant rated power output and to minimize structural loads. Generally, this is done by minimizing rotor speed deviations and damping tower and platform motion.
To realize the control goal of constant rated power production an additional state

$$
w=\int P_{e l} d t
$$

is introduced. In the linear model this yields

$$
\dot{w}=\frac{M_{g, S S}\left(v_{0, i}\right)}{i_{\text {gen }}} \Omega+\frac{\Omega_{S S}\left(v_{0, i}\right)}{i_{\text {gen }}} M_{g} .
$$

Translating the requests to the objective function $\Pi$ this gives

$$
\begin{aligned}
& \Pi=\boldsymbol{x}(k)^{T} \boldsymbol{Q} \boldsymbol{x}(k)+\boldsymbol{u}(k-1)^{T} \boldsymbol{R} \boldsymbol{u}(k-1)
\end{aligned}
$$

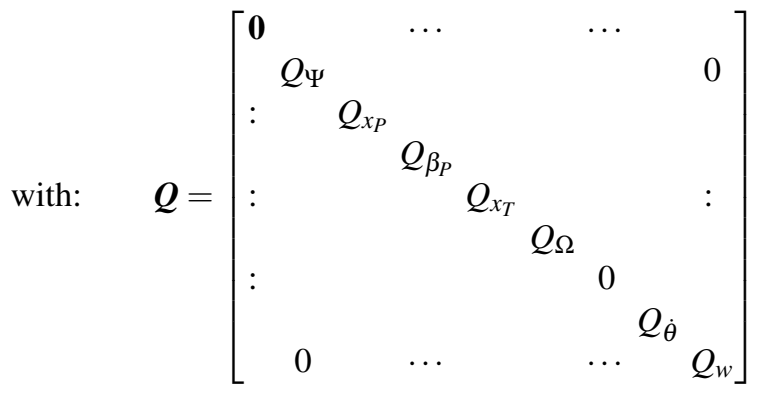

$$
\begin{aligned}
& \boldsymbol{R}=\left[\begin{array}{cc}
R_{M_{g}} & 0 \\
0 & R_{\theta_{c}}
\end{array}\right]
\end{aligned}
$$

To approximate the infinity cost of the optimal control problem a state-feedback controller is designed using the weighting matrices $\boldsymbol{Q}$ and $\boldsymbol{R}$ in a linear-quadratic regulator approach

$$
\Pi_{\infty}=\boldsymbol{x}(N)^{T} \boldsymbol{P} \boldsymbol{x}(N) .
$$

Furthermore, the operation of the FOWT is constrained to its operation range by a set of constraints $H$ which are

$$
\begin{aligned}
0^{\circ} & \leq \theta_{c} \leq 90^{\circ} \\
0.8 M_{g, S S} & \leq M_{g} \leq 1.2 M_{g, S S} \\
-8^{\circ} \mathrm{s}^{-1} & \leq \dot{\theta} \leq 8^{\circ} \mathrm{s}^{-1} .
\end{aligned}
$$

The optimal control problem is then comprised to a convex quadratic minimization problem with respect to the linear constraints and solved by a convex interior point method in Matlab. 
Below rated wind speed control In below rated wind conditions the main focus for the controller is to maximize power output. In current control strategies this is achieved as described in Section 4.1. Generally, the tip speed ratio $\lambda$ is controlled to be constant at its optimal value, $\lambda_{\text {opt }}$. To realize this in an optimal control approach, a new state is introduced

$$
\psi=\int \Omega-\Omega_{o p t} d t
$$

to realize an offset free tracking of the optimal rotor speed. $\Omega_{o p t}$ can be calculated assuming the current wind speed $v_{0}$ to be constant over the prediction horizon

$$
\Omega_{o p t}=\frac{\lambda_{o p t}}{R} v_{0}
$$

The second goal for the controller is to minimize structural loads. This is done by also penalizing the platform pitch motion and the tower fore-aft motion.

\section{Results}

In this section the presented controllers are evaluated within two simulation scenarios. First, an extreme operating gust (EOG) is presented to analyze the ability of damping the tower and platform motions. Second, the results of a one hour simulation using turbulent wind are compared. Finally, several statistical values of the simulations are compared to each other to confirm the observed results.

Extreme operating gust (EOG) Extreme operating gust simulations are mainly used to study extreme loads and the stability of the controller as well as the damping of the different motions. Here, an EOG at $14 \mathrm{~m} / \mathrm{s}$ is used. In Figure 9 the results are presented. The higher damping of the tower top motion $\dot{x}_{T}$ and the platform pitch motion $\dot{\beta}_{P}$ is observable. Further, the MPC controller has a higher performance in controlling the rotor speed and the generator power. This can be seen in evaluating the overshoot of both controller presented in Table 4 .

Turbulent wind simulation Besides extreme events, a controller for a FOWT has to guarantee a good performance in normal operation. To evaluate this ability simulations with a one hour turbulent wind field is proceeded. The results are presented in Figure 10. Further, some statistical values are evaluated in Table 4. The observations, namely a higher damping in tower top and platform pitch motion and good performance in stabilizing the rotor speed, made in the EOG simulations, can also be noticed in normal operation.
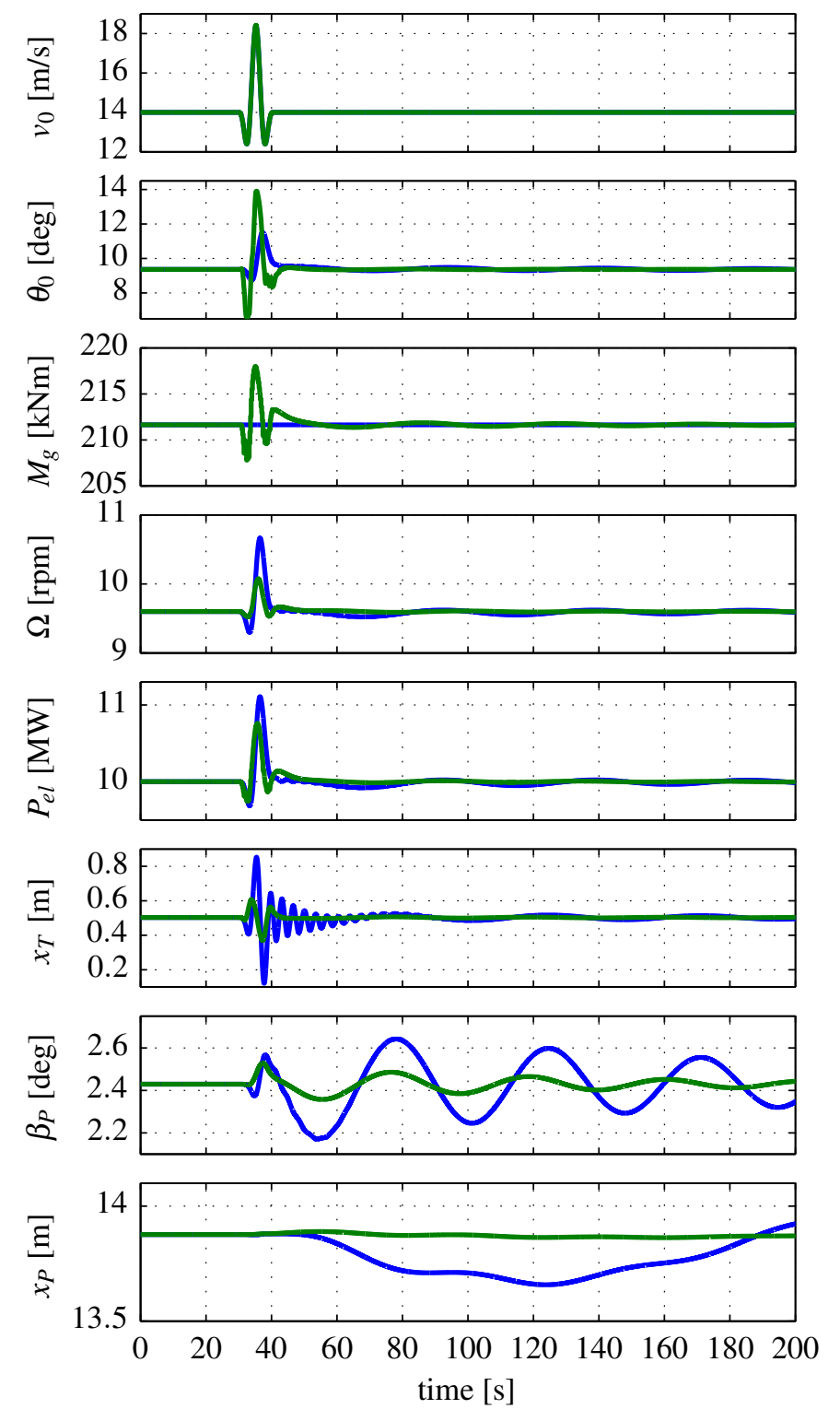

FIGURE 9: Comparisons of the different controllers at an EOG with $14 \mathrm{~m} / \mathrm{s}$. MPC (green), PI (blue).

\section{Conclusions and Outlook}

In this work a conceptual concrete platform model together with a 10MW wind turbine has been used for a study on the applicability of a linear model predictive controller (MPC) at an early conceptual design stage. A simplified coupled simulation model has been used with limited information on the aerodynamic blade characteristics. The model has been linearized and used for subsequent controller design. Besides the MPC a baseline PI-controller was developed as a reference, applying con- 
TABLE 4: Statistical values for both controllers in different operating situations.

\begin{tabular}{llccc} 
& & & PI & MPC \\
\hline EOG & overshoot $\frac{\Omega_{\max }}{\Omega_{\text {rated }}}$ & {$[-]$} & 1.11 & 1.05 \\
& overshoot $\frac{P_{\text {max }}}{P_{\text {rated }}}$ & {$[-]$} & 1.11 & 1.08 \\
& overshoot $\frac{x_{T, \text { max }}}{x_{T, S S}}$ & {$[-]$} & 1.70 & 1.21 \\
normal & $\sigma\left(\dot{x}_{T}\right)$ & {$[\mathrm{m} / \mathrm{s}]$} & 0.024 & 0.011 \\
operation & $\sigma\left(\dot{\beta}_{P}\right)$ & {$[\mathrm{deg} / \mathrm{s}]$} & 0.16 & 0.15 \\
& $\sigma(\Omega)$ & {$[\mathrm{rpm}]$} & 0.69 & 0.16 \\
& $\sigma(P)$ & {$[\mathrm{MW}]$} & 0.74 & 0.26 \\
\hline
\end{tabular}

ventional SISO methods using the same linear model. The linear MPC was developed and tuned to control the pitch and generator torque to minimize structural loads and to guarantee constant power output above rated wind speed. The concept of a linear MPC can be a good solution in facing the problems which arise in controlling a FOWT. With MPC it is straightforward to develop a well tuned optimal controller, which can be very helpful to obtain a target performance during the entire design phase of the FOWT system.

Altogether, both controllers show a satisfying performance, although the MPC damps the tower top and platform pitch motion better than the PI-controller and it reduces significantly the overshoot of the rotor speed compared to the PI-controller.

The concept of floating concrete foundations for large offshore wind turbines will be further investigated in the project INNWIND.EU.

Acknowledgements Parts of the research leading to these results has received funding from the European Communitys Seventh Framework Programme FP7-ENERGY-2012-1-2STAGE under grant agreement No. 308974 (INNWIND.EU).

\section{REFERENCES}

[1] Molins, C., Campos, A., Sandner, F., and Matha, D., 2014. "Monolithic concrete off-shore floating structure for wind turbines". In Proceedings of the EWEA.

[2] Veen, G. V. D., Couchman, Y., and Bowyer, R., 2012. "Control of floating wind turbines". In Proceedings of the American Control Conference ACC, pp. 3148-3153.

[3] Jonkman, J., 2008. "Influence of control on the pitch damping of a floating wind turbine". In Proceedings of the ASME Wind Energy Symposium.

[4] Larsen, T. J., and Hanson, T. D., 2007. "A method to avoid negative damped low frequent tower vibrations for a floating, pitch controlled wind turbine". Journal of Physics: Conference Series, 75.

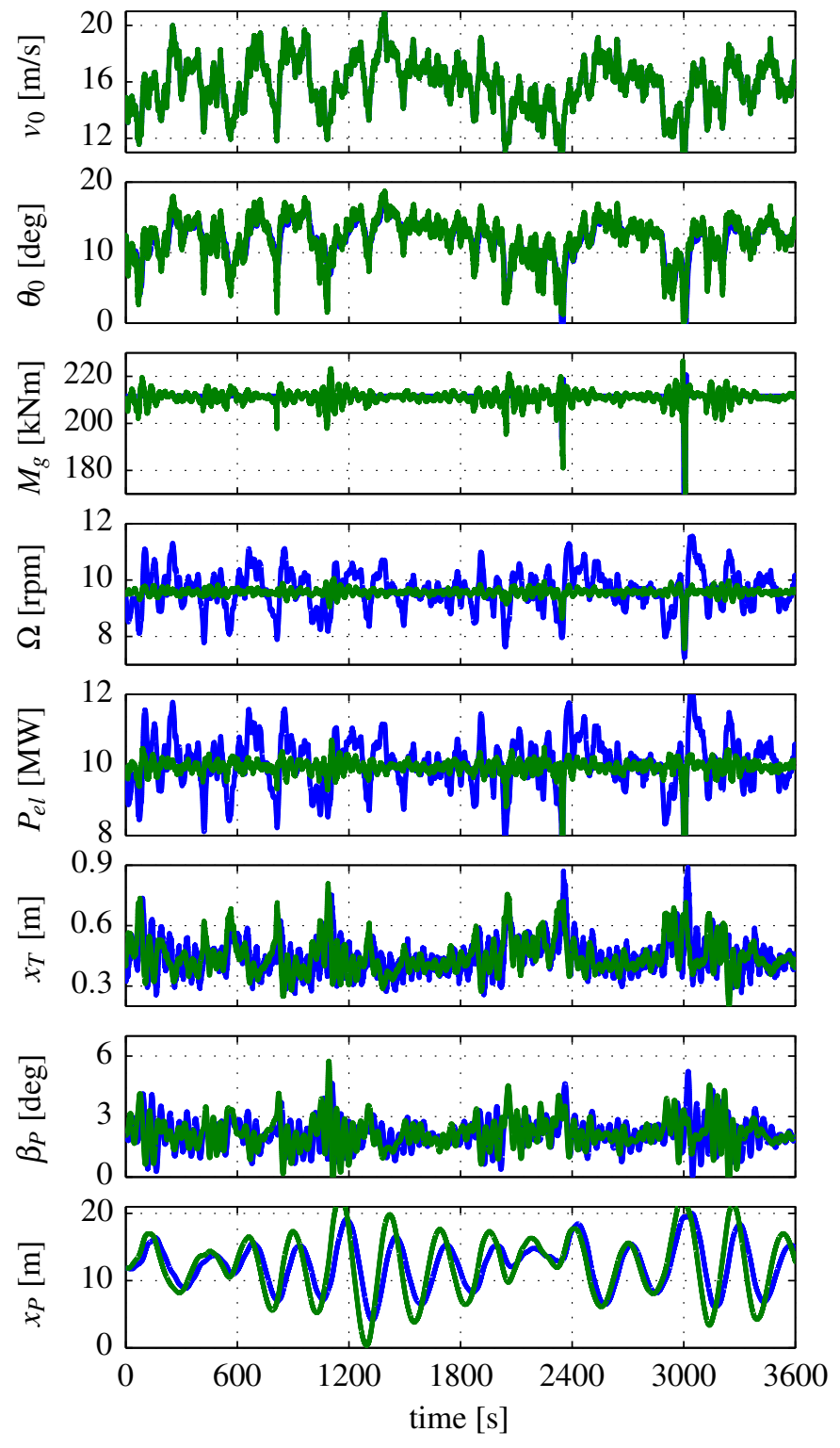

FIGURE 10: Comparison of baseline and model predictive controller with a turbulent wind field with $16 \mathrm{~m} / \mathrm{s}$ mean wind speed and no waves. MPC (green), PI (blue).

[5] Fleming, P., Pineda, I., Rossetti, M., Wright, A., and Arora, D., 2014. "Evaluating methods for control of an offshore floating turbine". In Proceedings of the ASME 2014 33rd International Conference on Ocean, Offshore and Arctic Engineering.

[6] Savenije, F., and Peeringa, J., 2014. "Control development for floating wind". Journal of Physics: Conference Series, 524, June, p. 012090. 
[7] Sandner, F., Schlipf, D., Matha, D., and Cheng, P. W., 2014. "Integrated optimization of floating wind turbine systems". In Proceedings of the 33rd International Conference on Ocean, Offshore and Arctic Engineering OMAE.

[8] Fischer, B., 2012. "Reducing rotor speed variations of floating wind turbines by compensation of non-minimum phase zeros". In Proceedings of the EWEA, pp. 144-147.

[9] Lackner, M., and Rotea, M., 2011. "Structural control of floating wind turbines". Mechatronics, 21(4), June, pp. 704-719.

[10] Christiansen, S., 2013. "Model based control of a ballast stabilized floating wind turbine exposed to wind and waves". PhD thesis, Aalborg University.

[11] Luo, N., Bottasso, C., Karimi, H. R., and Zapateiro, M., 2011. "Semiactive control for floating offshore wind turbines subject to aero-hydro dynamic loads". In International Conference on Renewable Energies and Power Quality.

[12] Namik, H., 2012. "Individual blade pitch and disturbance accommodating control of floating offshore wind turbines". $\mathrm{PhD}$ thesis, University of Auckland.

[13] Schlipf, D., Sandner, F., Raach, S., Matha, D., and Cheng, P. W., 2013. "Nonlinear model predictive control of floating wind turbines". In Proceedings of the Twenty-third International Conference on Offshore and Polar Engineering (ISOPE).

[14] Raach, S., Schlipf, D., Sandner, F., Matha, D., and Cheng, P. W., 2014. "Nonlinear model predictive control of floating wind turbines with individual pitch control". In Proceedings of the American Control Conference.

[15] Lindeberg, E., 2009. "Optimal control of floating offshore wind turbines”. Master's thesis, Norwegian University of Science and Technology.

[16] Bak, C., Zahle, F., Bitsche, R., Kim, T., Yde, A., Henriksen, L., Andersen, P., Natarajan, A., and Hansen, M., 2015. "Design and performance of a $10 \mathrm{mw}$ wind turbine (to be accepted)". Journal of Wind Energy.

[17] Jonkman, J., 2007. "Dynamics modeling and loads analysis of an offshore floating wind turbine". PhD thesis, National Renewable Energy Laboratory, Nov.

[18] Fossen, T., 2011. Handbook of Marine Craft Hydrodynamics and Motion Control, Vol. First Edit. John Wiley \& Sons.

[19] Lupton, R., and Langley, R., 2013. "Efficient modelling of floating wind turbines". In Proceedings of 9th PhD Seminar on Wind Energy in Europe.

[20] Karimirad, M., 2011. "Stochastic dynamic response analysis of spar-type wind turbines with catenary or taut mooring systems". PhD thesis, NTNU.

[21] Sandner, F., and Cheng, P. W., 2013. "Conceptual design of floating wind turbines". In Proceedings of the 9th $\mathrm{PhD}$ Seminar on Wind Energy in Europe.

[22] Matha, D., Sandner, F., and Schlipf, D., 2012. "Efficient critical design load case identification for floating offshore wind turbines with a reduced nonlinear model". The Science of Making Torque from Wind.

[23] Schiehlen, W., and Eberhard, P., 2004. Technische Dynamik - Modelle für Regelung und Simulation (in German). B.G. Teubner, Stuttgart.

[24] Burton, T., Jenkins, N., Sharpe, D., and Bossanyi, E., 2011. Wind Energy Handbook, 2nd ed. Wiley, Oxford.

[25] Soltani, M. N., Knudsen, T., Svenstrup, M., Wisniewski, R., Brath, P., Ortega, R., and Johnson, K., 2013. "Estimation of rotor effective wind speed: A comparison". IEEE Transactions on Control Systems Technology, 21(4), July, pp. 1155-1167. 